\title{
A Wearable Activity Sensor System and Its Physical Activity Classification Scheme
}

\author{
Fang-Chen Chuang ${ }^{1}$, Jeen-Shing Wang ${ }^{1}$, Ya-Ting Yang ${ }^{2}$, and Tzu-Ping Kao ${ }^{1}$ \\ ${ }^{1}$ Department of Electrical Engineering \\ ${ }^{2}$ Institute of Education \\ National Cheng Kung University \\ Tainan 701, Taiwan, R.O.C. \\ jeenshin@mail.ncku.edu.tw
}

\begin{abstract}
This paper presents a wearable activity sensor system and a systematic activity classification scheme for the classification of human daily physical activities. The wearable activity sensor system, consisting of two activity sensor modules worn on users' dominant hand wrists and ankles, is used for collecting activity acceleration signals. The proposed activity classification scheme, including static/dynamic activity analysis, posture recognition, exercise classification, and ambulation classification, is capable of classifying time-series activity acceleration signals. The collected acceleration signals are classify into two categories by means of static/dynamic activity analysis. Posture recognition is applied for partitioning static signals into sitting and standing. Exercise classification and ambulation classification algorithms were used to classify dynamic activity signals. Our experimental results have successfully validated the effectiveness of the proposed wearable sensor system and the scheme of activity classification algorithms with an overall classification accuracy of $96 \%$ for seven types of daily activities.
\end{abstract}

Keywords-physical activity classification; posture recognition; accelerometer; $\boldsymbol{k}$-nearest neighbor classification.

\section{INTRODUCTION}

The assessment of human daily physical activity requires reliable techniques and devices which are suitable for daily use. Over the past decade, numerous studies have developed physical activity monitoring and classification techniques using body-fixed sensor data [1], [2]. Due to the advantages of small size, portability, low power consumption, and low cost, accelerometers have become popular tools for monitoring human activities [3]. They have also been used to monitor a wide range of different human movements, measure physical activity levels, and classify physical activities [4].

Different computational techniques [1], [2], [5] have been applied successfully to the classification of physical activities using signals generated from accelerometers. Most classification approaches using accelerometers involve multistage procedures. In general, sensor signals are first divided into a number of small windows by a windowing process. Features, including time-domain, frequency-domain, and timefrequency features (or wavelet analysis), are then generated in order to evaluate the characteristics of sensor signals for each window. These features are used as inputs for classification algorithms which map each window to a specific physical activity. The degree of complexity required by different classification algorithms varies from relatively simple decision tree techniques to advanced algorithms, such as artificial neural networks (ANNs) or hidden Markov models (HMMs). However, existing activity classification schemes seldom applied divide-and-conquer strategies for classification of various physical activities ranging from sedentary to vigorous levels of intensity. Therefore, these activity classification schemes have not achieved satisfactory classification accuracy across different physical activity levels.

Many researchers have designed various sensors for collecting activity signals. For example, Preece et al. [6] proposed a sensor system simultaneously using three Pegasus activity monitors (attached to the participant's waist, thigh, and ankle) in order to achieve high classification accuracy. Each activity monitor contained a triaxial accelerometer. Timefrequency features were evaluated by a $k$-NN classifier to classify two groups of activities. The first group includes level walking, stair ascent, and stair descent, while the second group includes level walking, walking upstairs, walking downstairs, jogging, running, hopping on the left leg, hopping on the right leg, and jumping. Average classification accuracies using timefrequency features were $93.43 \%$ and $87.29 \%$ for the first and second groups respectively. Parkka et al. [4] placed sensors, consisting of a triaxial accelerometer and a triaxial compass, on participants' chests and wrists. In their experiment, participants were asked to maintain static postures (standing, sitting, and lying down) or engage in dynamic activities (walking, running, and cycling). The authors compared the classification accuracies of custom decision trees, and automatically generated decision trees. The average classification accuracies are $82 \%, 86 \%$, and $82 \%$, respectively.

In this study, we proposed a wearable activity sensor system and a physical activity classification scheme for classifying seven types of daily physical activities. The philosophy of the proposed physical activity classification scheme is to apply a divide-and-conquer strategy for first differentiating dynamic activities (e.g. walking, running, cycling, etc.) from static activities (e.g. sitting and standing), and then further classifying these two different categories of activities separately. The proposed scheme contains the following procedures: acceleration acquisition, signal preprocessing, static/dynamic activity analysis, posture 
recognition, exercise classification, and ambulation classification. The signal pre-processing procedure consists of high-pass filtering and windowing. A high-pass filter was used to filter gravitational accelerations from the raw data of the recorded acceleration signals. The pre-processed accelerations were divided into two categories, static and dynamic accelerations, by static/dynamic activity analysis. The procedure of posture recognition was used to discriminate sitting and standing postures for static accelerometer data. Features were then extracted from pre-processed dynamic accelerometer data, including count, standard deviation (STD), interquartile range (IQR), mean absolute deviation (MAD), norm, ratio of stance phase (RSP), skewness, kurtosis, the first, second, and third quartile (Q1, Q2, and Q3), energy, and entropy. Finally, exercise classification and ambulation classification classified five dynamic activity categories using $k$-nearest neighbor $(k$-NN) classifiers.

The remainder of this paper is organized as follows. In Section II, we introduce the hardware circuit of the wearable activity sensor system in detail. Alternative physical activity classification methods and the proposed physical activity classification scheme are presented in Section III. In Section IV, experimental results are presented to validate the effectiveness of the proposed approach and provide comparisons of classification accuracies for different physical activity classification methods. Conclusions are provided in the final section.

\section{WeARABLE ACTIVITY SENSOR SYSTEM}

The wearable activity sensor system is composed of a number of modules (shown in Fig. 1) to form a body motion network for sensing and recording body motion acceleration. Each module consists of a triaxial accelerometer (MMA7445L, Freescale ${ }^{\circledR}$ ), an MCU module (Microchip PIC24FJ64GA002), a wireless communication module (Nordic nRF2401+ and Bluetooth), and a storage module (16 MB flash memory). The dimensions of the sensor module, illustrated in Fig. 1, are 3.9 $\mathrm{cm} \times 3.3 \mathrm{~cm} \times 0.5 \mathrm{~cm}$. The triaxial accelerometer (MMA7445L) is responsible for sensing body motions and generating the acceleration signals for the corresponding motions. The accelerometer possesses a full scale selection of $\pm 2 \mathrm{~g}, \pm 4 \mathrm{~g}$, and $\pm 8 \mathrm{~g}$, and is able to measure accelerations over the bandwidth of $125 \mathrm{kHz}$ for all axes. In this study, the accelerometer's sensitivity was set from $-8 \mathrm{~g}$ to $+8 \mathrm{~g}$. The Microchip $\mathbb{R}$ PIC24FJ64GA002 was selected as the MCU module and was responsible for the following tasks: 1) timing flow control for regularly retrieving accelerometer data from the sensor module via an $\mathrm{I}^{2} \mathrm{C}$ bus, 2) converting analog acceleration signals into digital signals via its embedded A/D converter, 3) wireless communication control and module synchronization, and 4) peripheral component control. The wireless communication module includes a Nordic ${ }^{\circledR}$ nRF24L01+ wireless RF transceiver and a BTM401 Bluetooth ${ }^{\circledR}$ module. The Nordic $\AA$ nRF24L01+ wireless RF transceiver is used to execute timing synchronization and data transmission among sensor modules worn on different positions of a human body. The BTM401 Bluetooth ${ }^{\circledR}$ module serves as the standard communication channel between the host of the body sensor network and standardized devices such as PCs or smart phones. The storage module employed in the sensor module is an MXIC®
MX25L128 flash memory with a 16 MB storage capacity. A Microchip ${ }^{\circledR}$ PIC24FJ64GA002 main controller is selected to maximize the performance and minimize the power consumption of the sensor module. The sampling rate $(f s)$ of the sensor module is $30 \mathrm{~Hz}$ with 12-bit data resolution, while power consumption is $12.2 \mathrm{~mA}$ at $4.2 \mathrm{~V}$. A schematic diagram of the sensor module is shown in Fig. 2.

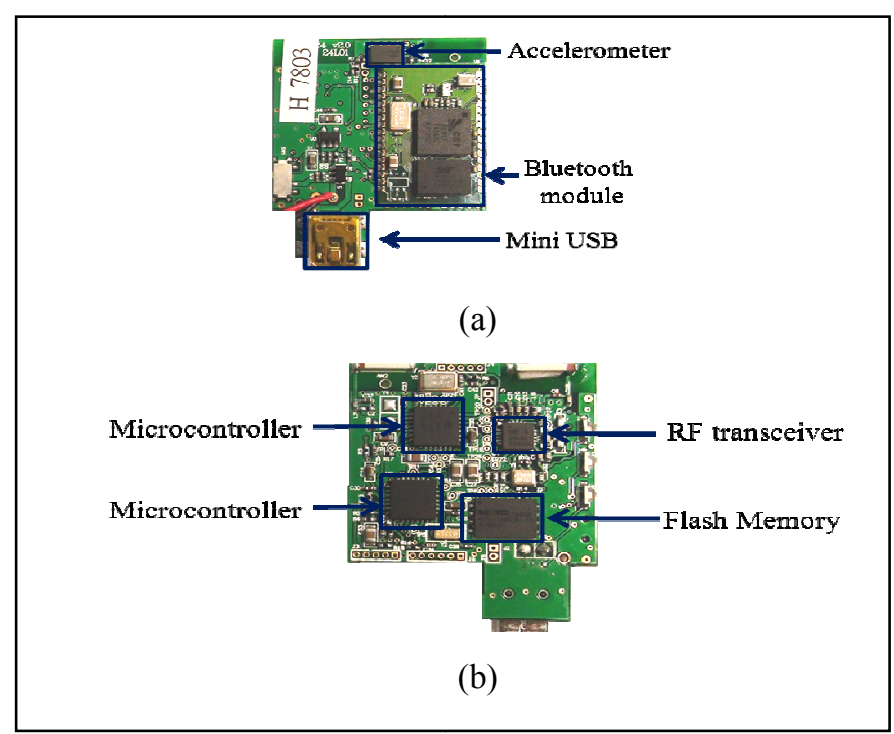

Figure 1. The circuit board of the wearable activity sensor module: (a) front view and (b) back view.

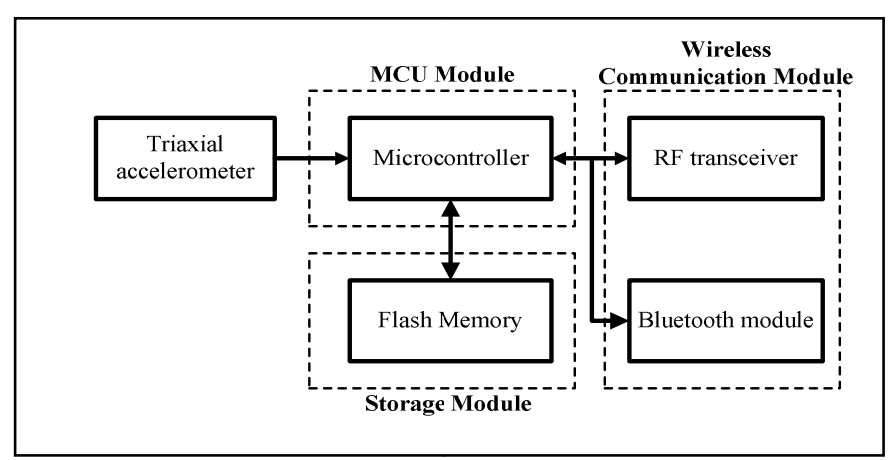

Figure 2. Schematic diagram of the wearable activity sensor module.

\section{Physical ACTIVITY ClassificATION SCHEME}

Recent studies introduced in Section I have mentioned that most classification approaches using accelerometers usually involve multi-stage procedures. First, sensor signals are divided into a number of small windows by a windowing process, and then features are generated in order to evaluate the characteristics of sensor signals for each window. Finally, these features are used as inputs for different classification algorithms, such as the $k$-NN algorithm [6], [7] and ANNs [4], [7], etc. We adopted the same classification strategy to develop two activity classification methods for various physical activities ranging from sedentary to vigorous intensity levels. The activity classification methods are described in the following sub-section A. However, the acceleration signals of static activities do not vary obviously. This phenomenon 
causes that features generated from acceleration signals of static activities are too similar, and thus these features of static activities are not helpful to discriminate sitting and standing activities from each other. In order to improve classification accuracy, the proposed physical activity classification scheme is to apply a divide-and-conquer strategy by differentiating dynamic activities (e.g. walking, running, cycling, etc.) from static activities (e.g. sitting and standing). Then, posture recognition was used to classify static activities to enhance the classification accuracy. In the dynamic activities, the exercise classification algorithm was first used to classify running, cycling, and ambulation activities. Subsequently, the ambulation classification algorithm was utilized to classify ambulation activities including level walking, walking downstairs, and walking upstairs. The proposed physical activity classification scheme is described in the sub-section B.

\section{A. Two Activity Classification Methods}

The block diagrams of Methods I and II are illustrated in Fig. 3(a) and (b). Methods I and II have the same steps, consisting of acceleration acquisition, signal pre-processing, feature generation, linear discriminant analysis (LDA), and classifier construction. Participants were asked to attach two activity sensor modules to their wrists and ankles to collect the acceleration signals of physical activities.

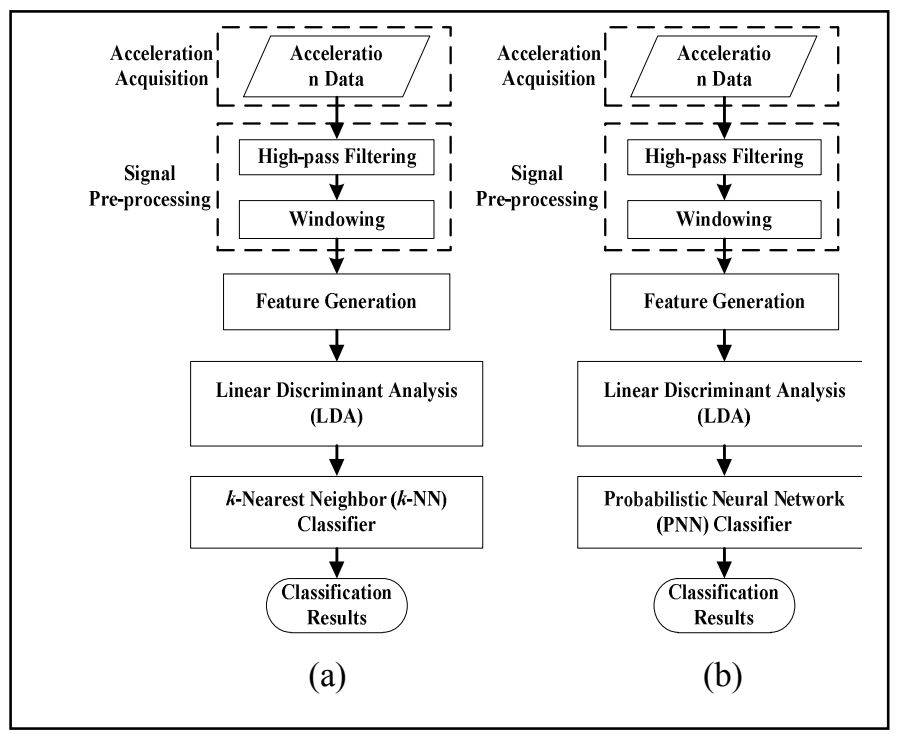

Figure 3. Flowcharts for (a) Method I and (b) Method II.

These acceleration signals were first pre-processed by filtering and windowing procedures. Features were then generated from the windowed signals according to the signal characteristics of different activities. After feature generation, the LDA was utilized to reduce the dimension of the generated features. Finally, a $k$-NN classifier and a probabilistic neural network (PNN) classifier were constructed for Methods I and II, respectively.

\section{- Signal Pre-processing}

The sensor system records accelerations, including both gravitational acceleration and body acceleration (BA). A highpass filter is required to remove gravitational acceleration and obtain the BA component after calibration. In general, it is difficult to analyze and recognize activities from a long-term and continuous sequence of acceleration data. In the data preprocessing procedure, accelerations are segmented into nonoverlapping windows of 5 seconds in length (150 sample points) and signal vector magnitude (SVM) [8] as (1) is calculated by

$$
\operatorname{SVM}(n)=\sqrt{A c c_{x_{n}}{ }^{2}+A c c_{y_{n}}{ }^{2}+A c c_{z_{n}}{ }^{2}}
$$

where $A c c_{x_{n}}, A c c_{y_{n}}$, and $A c c_{z_{n}}$ are the $n$th sample of the $x$-, $y$-, and $z$-axis acceleration signals generated by the sensor activity module.

\section{- Feature Generation}

Characteristics of acceleration signals from different physical activities were obtained by extracting features from preprocessed SVM signals that were used to generate thirteen features: 1) eleven time-domain features: count [9], STD, IQR [6], MAD [5], norm [10], RSP, skewness, kurtosis [11], Q1, Q2, and Q3 [12], and 2) two frequency-domain features: energy [5] and entropy [13]. A total of 25 features were generated from the feature generation process. The feature vectors were then used for activity classification methods.

\section{- Linear Discriminant Analysis (LDA)}

For pattern recognition problems, LDA [14] is an effective dimensionality reduction method which uses a linear transformation to transform the original feature sets into a lower dimensional feature space. The purpose of LDA is to divide the data distribution in different classes and minimize the data distribution of the same class in a new space.

\section{- Classifiers Construction}

\section{(1) $k$-NN Classifier Construction for Method I}

The $k$-NN [15] is one of the low-computation complexity methods for pattern recognition, and the principle of $k-\mathrm{NN}$ is based on an intuitive concept that data points of the same class should be closer in the feature space. To specify $k$-NN more rigorously, a given training dataset of $n$ points with their desired class is supposed as $\left\{\left(\mathbf{x}_{1}, y_{1}\right),\left(\mathbf{x}_{2}, y_{2}\right), \ldots,\left(\mathbf{x}_{n}, y_{n}\right)\right\}$, where $\left(\mathbf{x}_{i}, y_{i}\right)$ represents data pair $i, \mathbf{x}_{\mathrm{i}}$ is the feature vector, and $y_{i}$ is the corresponding target class. Then for a new data point $\mathbf{x}$, the most likely class should be determined by $k$-NN $(k=1$ in this case):

$$
1-\mathrm{NN}(\mathbf{X})=y_{p}
$$

where

$$
p=\arg \min _{i}\left|\mathbf{x}-\mathbf{x}_{i}\right|^{2}
$$

The above equation uses the nearest neighbor to label the data class.

(2) Probabilistic Neural Network (PNN) Classifier Construction for Method II

The PNN first proposed by Specht [16] was employed as the classifier in this study. With enough training data, the PNN 
is guaranteed to converge to a Bayesian classifier, and thus it has a great potential for making classification decisions accurately and providing probability and reliability measures for each classification. In addition, the training procedure of the PNN needs only one epoch to adjust the weights and biases of the network architecture. Therefore, the most important advantage of using the PNN is its high speed of learning. Typically, the PNN consists of an input layer, a pattern layer, a summation layer, and a decision layer. The output of the PNN was represented by the label of the desired outcome defined by researchers. For example, in this study, the labels ' 1 ' was used to represent activity category 1 .

\section{B. Physical Activity Classification Scheme}

The block diagram of the proposed classification scheme is illustrated in Fig. 4.

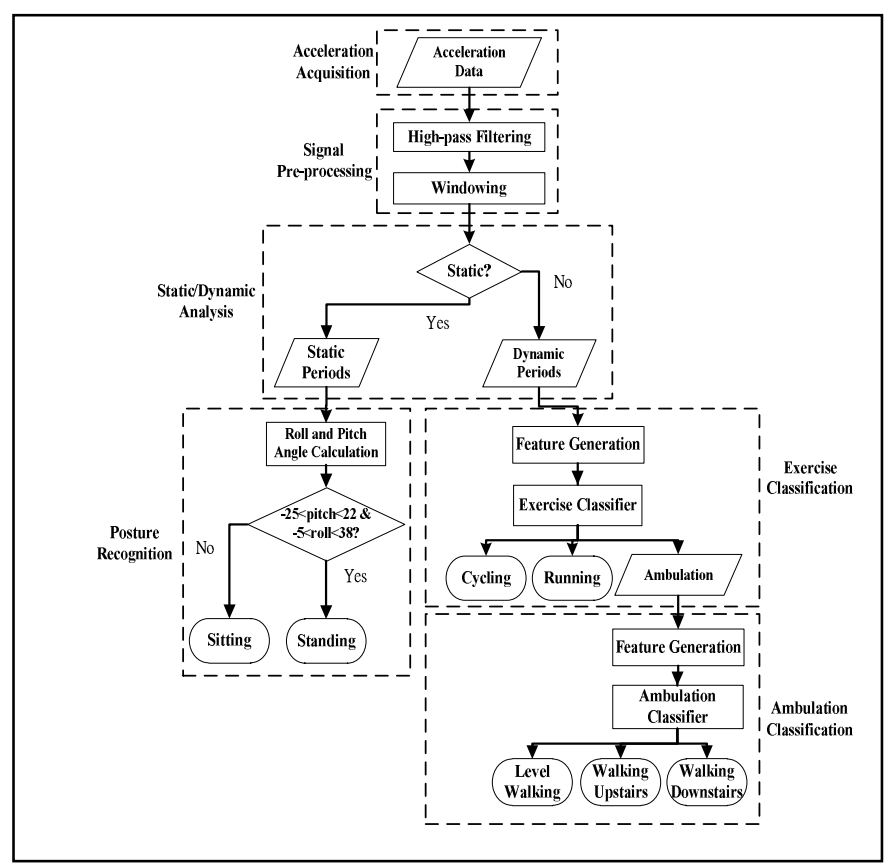

Figure 4. Flowchart of the proposed physical activity classification algorithm.

The proposed classification scheme consists of the processes of acceleration acquisition, signal pre-processing, static/dynamic activity analysis, posture recognition, exercise classification, and ambulation classification. Participants wore two activity sensor modules to collect the acceleration signals of physical activities, and the acceleration signals were then pre-processed by filtering and windowing procedures. The procedure of the signal pre-processing has been described in the above section. Static/dynamic activity analysis was used to first differentiate dynamic activities from static activities. Subsequently, static periods were divided into sitting and standing categories by posture recognition. For dynamic periods, thirteen features were first generated from the windowed signals according to the signal characteristics of different activities, and the process of feature generation has been described in the above section. Then, a $k$-NN classifier was trained for exercise classification, while another $k$-NN classifier was applied for ambulation classification. The detailed procedures of the proposed physical activity classification scheme are introduced as follows.

\section{- Static/dynamic Activity Analysis}

The purpose of static/dynamic activity analysis is to discriminate between static and dynamic activity periods. We transform continuous acceleration signals of each window into a discrete value named "count" in order to determine whether each windowed signal belongs to "static" or "dynamic" periods. Here, we define the "static" period as intervals during which participants' feet are not moving. According to our empirical analysis, the threshold of the count value for determining static/dynamic activity periods was set at 0.12 . That is, if the count for a given window was larger than 0.12 , this windowed signal was defined as a "dynamic" period. Conversely, windows of less than 0.12 were defined as "static".

\section{- Posture Recognition}

After the static/dynamic activity analysis, we aim to provide a distinction between sitting and standing positions using posture recognition. Euler angles, an orientation representation method, are adopted to obtain the pitch angle $(\theta)$ and the roll angle $(\phi)$ :

$$
\begin{aligned}
& \theta=\tan ^{-1} \frac{\sum_{n=1}^{N} A c c_{x}(n)}{\sum_{n=1}^{N} A c c_{y}(n)}, \\
& \phi=\tan ^{-1} \frac{\sum_{n=1}^{N} A c c_{z}(n)}{\sum_{n=1}^{N} A c c_{y}(n)},
\end{aligned}
$$

where $A c c_{x}, A c c_{y}$, and $A c c_{z}$ are the same as those described in (1), and $N$, representing the window size, is equal to 150 .

In general, when people stand without leaning against any object, their position is nearly vertical, such that pitch and roll angles for the ankle will be near $0^{\circ}$. On the contrary, the legs are tilted during sitting on a chair leisurely. Therefore, pitch and roll angles can be used to determine sitting and standing postures. According to our empirical study, the pitch angles between $-25^{\circ}$ and $22^{\circ}$ and the roll angles between $-5^{\circ}$ and $38^{\circ}$ were defined as "standing", while all other angles were categorized as "sitting".

\section{- Exercise Classification and Ambulation Classification}

In this study, exercise classification was first developed for classifying running, cycling, and ambulation activities which include level walking, walking upstairs, and walking downstairs. Subsequently, ambulation classification was proposed to focus on the walking patterns classification. Exercise and ambulation classification required the processes of feature generation and the construction of activity classifiers. Here, features which have been introduced in the above section were generated from pre-processed signals to represent the characteristics of different activities, while the first and second $k$-NN classifiers were used for exercise and ambulation classification, respectively. Here, the parameter $k$ of the two classifiers was set as 5 .

\section{- Summary of Physical Activity Classification Scheme}

We now summarize the proposed physical activity classification scheme development process in the following steps. 
Step 1: Acquire raw acceleration signals from two sensor modules.

Step 2: Remove gravity acceleration from filtered accelerations using a high-pass filter, and then segment acceleration data into non-overlapping windows of 5 seconds in length by a windowing process.

Step 3: Discriminate periods using static/dynamic activity analysis.

Step 4: Recognize each static period as either "sitting" or "standing" using posture recognition.

Step 5: Generate time- and frequency-domain features from SVM signals for dynamic activities.

Step 6: Classify five dynamic activity categories including running, cycling, level walking, walking upstairs, and walking downstairs using $k$-NN classifiers.

\section{EXPERIMENTAL RESULTS}

The effectiveness of the proposed physical activity classification scheme was validated for seven categories of physical activity. Acceleration signals were collected from ten subjects. Participant demographic information and experimental procedures will be introduced.

\section{A. Participants}

Ten participants, aged 20 to 25 years old, with an average body mass index (BMI) of $21.78 \pm 2.82 \mathrm{~kg} / \mathrm{m}^{2}$ were recruited to participate in this experiment. All participants were nonsmokers, disease-free, and not currently taking medications known to influence metabolic rates.

\section{B. Experimental Procedures}

All participants were asked to complete various lifestyle activities and exercises including the following seven categories.

1) Category 1 (sitting): doing computer work and wiping the ground with a dry cloth.

2) Category 2 (standing): sweeping, washing dishes, cleaning tables, and picking up objects.

3) Category 3 (upstairs): walking upstairs.

4) Category 4 (downstairs): walking downstairs.

5) Category 5 (level walking): walking at a normal pace on a sports field.

6) Category 6 (running): running on a sports field.

7) Category 7 (cycling): riding a bicycle on a sports field.

Before this experiment, each participant was instructed to wear a sensor module mounted on both wrist and ankle. Participants performed each activity for 3 minutes, and took a rest between two activities to ensure their heart rates were below $100 \mathrm{bpm}$. Acceleration signals from the 3 minute activity periods were extracted for analysis. We segmented acceleration data into non-overlapping windows and performed static/dynamic activity analysis for each window.

\section{Experimental Results}

The proposed classification scheme separates dynamic activities from static activities before classifying these two different types of activities separately. This classification scheme consists of processes for acceleration acquisition, signal pre-processing, static/dynamic activity analysis, posture recognition, exercise classification, and ambulation classification. Feature generation and classifier construction were conducted for both exercise classification and ambulation classification. For the signals generated by static activities, we used posture recognition to classify sitting and standing positions. Using pre-processed acceleration data for dynamic activities, 25 features were generated using the feature generation procedure. Finally, exercise and ambulation classification algorithms were used to classify five dynamic activity categories.

A total of $3960(10 \times 11 \times 36)$ physical activities were evaluated. We adopted the leave-one-subject-out crossvalidation method to evaluate the proposed physical activity classification performance. That is, the physical activity classifier was trained with the generated dataset from all subjects except the data from one randomly selected participant, which was used as the test data. Hence, $396(1 \times 11 \times 36)$ samples were used for the testing dataset of the cross-validation procedure, while 3564 activity samples from the remaining participants $(9 \times 11 \times 36)$ were used as training data in each repetition of the cross-validation process. We repeated the procedure for all the subjects. The confusion matrix and classification accuracies for the proposed activity classification scheme are shown in Tables I and II. The range of classification accuracy was from 94.44 to $99.44 \%$, with an average classification accuracy of $96.82 \%$.

TABLE I. CONFUSION MATRIX OBTAINED BY THE PROPOSED PHYSICAL ACTIVITY CLASSIFICATION ALGORITHM

\begin{tabular}{|c|c|c|c|c|c|c|c|}
\hline $\begin{array}{c}\text { Classified } \\
\text { as }\end{array}$ & Sit & Stand & Up-stairs & $\begin{array}{c}\text { Down- } \\
\text { stairs }\end{array}$ & Walk & Run & Cycling \\
\hline Sit & 700 & 14 & 0 & 0 & 6 & 0 & 0 \\
\hline Stand & 54 & 1386 & 0 & 0 & 0 & 0 & 0 \\
\hline $\begin{array}{c}\text { Up- } \\
\text { stairs }\end{array}$ & 0 & 0 & 344 & 4 & 11 & 0 & 1 \\
\hline $\begin{array}{c}\text { Down- } \\
\text { stairs }\end{array}$ & 0 & 0 & 2 & 358 & 0 & 0 & 0 \\
\hline Walk & 0 & 0 & 5 & 15 & 340 & 0 & 0 \\
\hline Run & 0 & 0 & 0 & 2 & 0 & 358 & 0 \\
\hline Cycling & 5 & 0 & 0 & 7 & 0 & 0 & 348 \\
\hline
\end{tabular}

TABLE II. CONFUSION MATRIX OBTAINED BY THE PROPOSED PHYSICAL ACTIVITY CLASSIFICATION ALGORITHM

\begin{tabular}{|c|c|c|c|c|c|c|c|}
\hline & \multicolumn{7}{|c|}{ Accuracy (\%) } \\
\hline Methods & Sit & Stand & Up-stairs & $\begin{array}{c}\text { Down- } \\
\text { stairs }\end{array}$ & Walk & Run & Cycling \\
\hline $\begin{array}{c}\text { Our } \\
\text { Scheme }\end{array}$ & $\mathbf{9 7 . 2 2}$ & $\mathbf{9 6 . 2 5}$ & $\mathbf{9 5 . 5 6}$ & $\mathbf{9 9 . 4 4}$ & $\mathbf{9 4 . 4 4}$ & $\mathbf{9 9 . 4 4}$ & $\mathbf{9 6 . 6 7}$ \\
\hline Method I & 50.14 & 89.03 & 83.33 & 77.22 & 70.00 & 99.17 & 92.78 \\
\hline Method II & 40.83 & 94.03 & 83.06 & 78.89 & 73.89 & 99.44 & 93.33 \\
\hline
\end{tabular}

In addition, a comparison of classification accuracies between the proposed physical activity classification algorithm and the two classification methods is shown in Table II. The 
average classification accuracy obtained by the proposed classification algorithm was the best among the methods (96.82\%), with a classification rate above $94 \%$ for each category. Classification accuracies from $94.44 \%$ for "walking" to $99.44 \%$ for "walking downstairs" and "running" were observed. It should be noted that classification accuracies were between $40.83 \%$ and $50.14 \%$ for the sitting category and $70 \%$ to $73.89 \%$ of the walking category using Methods I and II. The performance of these methods for "sitting" and "walking" were considered poor. Hence, the overall classification rate for the four methods was reduced due to low classification accuracies for the "sitting" and "walking" categories, with overall classification accuracies for Methods I and II of $80.24 \%$ and $80.50 \%$, respectively. The proposed physical activity classification scheme can not only recognize static postures successfully but can also effectively classify dynamic physical activities ranging from light to vigorous intensity levels.

\section{CONCLUSIONS}

A scheme for the classification of daily physical activities using acceleration signals has been developed in this study. During the experiment, the acceleration signals were collected by two wearable activity sensor modules: one module was worn on participants' ankles and another was worn on their wrists. The proposed physical activity classification scheme, including static/dynamic activity analysis, posture recognition, exercise classification, and ambulation classification, was capable of classifying time-series activity acceleration signals. The scheme first divided acceleration signals into static and dynamic categories by means of static/dynamic activity analysis. Posture recognition was used to partition static observations into sitting and standing categories. Then, exercise classification and ambulation classification algorithms were used to classify dynamic activities. In this experiment, accelerations were collected for seven categories of physical activities to validate the effectiveness of the proposed physical activity classification scheme. The experimental results, with the overall classification accuracy of $96 \%$, confirm the effectiveness of the proposed approach. According to the experimental results, we can see that the proposed physical activity classification algorithm demonstrates satisfactory performance not only for recognizing static postures but also for classifying various physical activities ranging from light to vigorous levels of intensity. This result encourages us to further investigate the possibility of using the developed wearable activity sensor system as an effective tool for physical activity monitoring applications.

\section{REFERENCES}

[1] M. J. Mathie, A. C. F. Coster, N. H. Lovell, and B. G. Celler, "Accelerometry: Providing an integrated, practical method for long-term, ambulatory monitoring of human movement," Physiological Measurement, vol. 25, no. 2, pp. R1-R20, 2004.

[2] L. Dong, J. Wu, and X. Chen, "Real-time physical activity monitoring by data fusion in body sensor networks," in Proc. of $10^{\text {th }}$ IEEE Int'l Conf. on Information Fusion, 2007, pp. 1-7.

[3] D. M. Karantonis, M. R. Narayanan, M. Mathie, N. H. Lovell, and B. C. Celler, "Implementation of a real-time human movement classifier using a triaxial accelerometer for ambulatory monitoring," IEEE Trans. Information Technology in Biomedicine, vol.10, no.1, pp. 156-167, 2006.

[4] J. Parkka, M. Ermes, P. Korpipaa, J. Mantyjarvi, J. Peltola, and I. Korhonen, "Activity classification using realistic data from wearable sensors," IEEE Trans. Information Technology in Biomedicine, vol. 10, no. 1, 119-128, 2006.

[5] Y. P. Chen, J. Y. Yang, S. N. Liou, G. Y. Lee, and J. S. Wang, "Online classifier construction algorithm for human activity detection using a triaxial accelerometer," Applied Mathematics and Computation, pp. 849$860,2008$.

[6] S. J. Preece, J. Y. Goulermas, L. P. J. Kenney, and D. Howard, "A comparison of feature extraction methods for the classification of dynamic activities from accelerometer data," IEEE Trans. Biomedical Engineering, vol. 56, no. 3, pp. 871-879, 2009.

[7] P. Pirttikangas, K. Fujinami, and T. Nakajima, "Feature selection and activity recognition from wearable sensors," Lecture Notes in Computer Science, vol. 4239, pp. 516-527, 2006.

[8] D. M. Karantonis, M. R. Narayanan, M. Mathie, N. H. Lovrll, and B. G. Celler, "Implementation of a real-time human movement classifier using a triaxial accelerometer for ambulatory monitoring," IEEE Trans. Information Technology in Biomedicine, vol. 10, no. 1, pp. 156-167, 2006.

[9] K. Y. Chen and D. R. Bassett, "The technology of accelerometry-based activity monitors: Current and future," Medicine \& Science in Sports \& Exercise, vol. 37, no. 11, pp. S490-S500, 2005.

[10] Prugovecki, Eduard, Quantum Mechanics in Hilbert Space. Academic Press, 1981.

[11] R. A. Groeneveld and G. Meeden, "Measuring skewness and kurtosis", The Statistician, vol. 33, pp. 391-399, 1984.

[12] H. Rob and F. Yan, "Sample quantiles in statistical packages", American Statistician, vol. 50, no. 4, pp. 361-365, 1996.

[13] L. Bao and S. S. Intille, "Activity recognition from user-annotated acceleration data," Lecture Notes in Computer Science, pp. 1-17, 2004.

[14] A. M. Martinez and A. C. Kak, "PCA versus LDA", IEEE Trans. Pattern Analysis and Machine Intelligence, vol. 23, no. 2, pp. 228-233, 2001.

[15] T. M. Cover and P. E. Hart, "Nearest neighbor pattern classification", IEEE Trans. Information Theory, vol. 13, no. 1, pp. 21-27, 1967.

[16] D. F. Specht, "Probabilistic neural networks," Neural Networks, vol. 3, pp. 109-118, 1990. 